\title{
Achievable transmission capacity of cognitive radio networks with cooperative relaying
}

\author{
Tao Jing ${ }^{1 *}$, Wei Li ${ }^{2}$, Xiuying Chen ${ }^{1}$, Xiuzhen Cheng ${ }^{2}$, Xiaoshuang Xing ${ }^{2}$, Yan Huo ${ }^{1}$, Tao Chen ${ }^{3}$, \\ Hyeong-Ah Choi $^{2}$ and Taieb Znati ${ }^{4}$
}

\begin{abstract}
With the rapid development of cognitive radio technologies, spectrum sharing becomes a promising approach to improving the efficiency of spectrum utilization and mitigating the spectrum scarcity problem. Previous research on cognitive networks argues that secondary users can only work under a low-transmission power in an underlay spectrum sharing model, especially when the primary transmitter is far away from the primary receiver. Motivated by the idea of cooperative communications, in this paper, we propose a cooperative framework in which a primary transmitter, being aware of the existence of the secondary network, may select a secondary user that is not in transmitting or receiving mode to relay its traffic. The feasible relay location region and optimal power ratio between the primary network and the secondary network are derived in the underlay spectrum sharing model. Based on the optimal power ratio, we derive the maximum achievable transmission capacity of the secondary network under the outage constraints from both the primary and the secondary network with or without cooperative relaying. Numerical results indicate that secondary users can achieve a higher transmission capacity with cooperative relaying, and that the capacity gain of the cooperative network is significantly affected by the location of the relay and the network system parameters.
\end{abstract}

Keywords: Achievable transmission capacity; Cognitive radio networks; Cooperative relaying; Outage constraints

\section{Introduction}

Due to the rapid development of wireless communications in recent years, the demand on wireless spectrum has been growing dramatically, resulting in the spectrum scarcity problem. Works have shown that the fixed spectrum allocation policy commonly adopted today suffers from the low spectrum utilization problem [1,2]. Both academic and regulatory bodies have focused on dynamic spectrum access aiming at enhancing the utilization of the spectrum resource. Cognitive radio [3-6], with the capability to flexibly adapt its parameters, has been proposed as the enabling technology for unlicensed secondary users (SUs) to dynamically access the licensed spectrum owned by legacy primary users (PUs) on a negotiated or an opportunistic basis.

*Correspondence: tjing@bjtu.edu.cn

${ }^{1}$ School of Electronics and Information Engineering, Beijing Jiaotong University, No. 3 Shangyuancun, Haidian District, Beijing 100044, People's Republic of China

Full list of author information is available at the end of the article
As a fundamental problem, the achievable transmission capacity of a cognitive radio network over the underlay spectrum sharing model has been extensively studied [7-9], in which the secondary users are allowed to access the channel only if their transmissions do not cause intolerable interference at the primary receiver. Our prior work [10] indicates that secondary users could only work at a low transmission power over the underlay spectrum sharing model to guarantee the normal communications of the primary users, especially when the direct transmission from a primary transmitter to its receiver is severely damaged due to path loss and channel fading; thus secondary users can successfully receive signals only with a low reception threshold, leading to a low transmission capacity. Motivated by the idea of cooperative relaying, in this paper, we aim to study whether cooperative communications can help the secondary users to achieve a higher transmission capacity constrained by the outage probabilities from both the primary and the secondary users compared to the cognitive network without cooperative relaying under the physical interference model, in which

\section{望 Springer}

(C) 2015 Jing et al: licensee Springer. This is an Open Access article distributed under the terms of the Creative Commons Attribution License (http://creativecommons.org/licenses/by/4.0), which permits unrestricted use, distribution, and reproduction in any medium, provided the original work is properly credited. 
a transmission is successful if and only if the corresponding signal-to-interference-plus-noise ratio (SINR) at the receiver is higher than a threshold.

Our work deviates from the current research in that most existing papers usually assume the mechanism in which primary users lease their spectrum to secondary users for a fraction of time and in exchange, they get cooperative transmissions or other benefits. In this paper, we investigate the achievable transmission capacity of a cooperative cognitive network over the underlay spectrum sharing model, in which a secondary user is allowed to use the primary spectrum simultaneously with the primary user as long as it does not undermine the successful transmissions of the primary system. Our main contributions are summarized as follows:

1. Though the cooperative framework for the overlay or interweave spectrum sharing model has been proposed, we lack a framework for cooperative relaying under the underlay spectrum sharing model, in which a primary user far away from its receiver can select a secondary user, which is not in transmitting or receiving mode and has a higher link quality, to relay its packets. We propose such a framework to ensure that secondary users can transmit over the same spectrum band as the primary user at the same time at a higher power without disturbing the communications of the primary network.

2. We obtain the successful transmission probabilities of the primary and the secondary network under the physical interference model. We also identify and validate the feasible location region of the relay for a primary transmitter and its receiver.

3. The maximum transmission capacities of a cognitive network with and without cooperative relaying are derived under the outage probability constraints from both the primary and the secondary network. Two relaying protocols, namely decode-and-forward (DF) and amplify-and-forward (AF), are considered in our analysis.

4. Numerical results are reported to illustrate our argument, which states that cognitive networks can achieve a higher transmission capacity when secondary users provide cooperative relaying for the primary network over the underlay model constrained by the outage probabilities of both the primary and the secondary system, and the capacity gain in cooperative relaying is significantly affected by the location of the relay and the network system parameters.

The rest of the paper is organized as follows. Related works are summarized in Section 2. Section 3 depicts our system model. In Section 4, we derive the achievable transmission capacity of the secondary network when no cooperative relaying is employed. Section 5 details the elaboration on the achievable transmission capacity with cooperative relaying for both the decode-and-forward and the amplify-and-forward relay protocol. Our numerical analysis is reported in Section 6, and Section 7 concludes the paper.

\section{Related work}

Basic cognitive radio systems can be divided into three paradigms $[2,11,12]$ : overlay, underlay, and interweave. In the overlay model, secondary users employ the same spectrum concurrently with primary users if the interference to the primary users can be offset via channel coding or network coding. In the underlay model, secondary users are allowed to access the channel only if their transmissions do not cause intolerable (accumulated) interference at the primary receivers. In the interweave model, secondary users can only access the spectrum where the primary users are not active, known as spectrum holes, through periodically monitoring the radio spectrum. Since the overlay model needs the facilitation of sophisticated signal process and coding techniques [11], and the interweave model requires secondary users to have high sensing accuracy, our research focuses on the underlay model, in which concurrent transmissions may occur between the secondary and primary users as long as the interference generated by the secondary users at the primary receivers is below a certain acceptable threshold.

In an underlay cognitive radio network, the achievable transmission capacity of the secondary network under the constraints of the outage probabilities of the primary and the secondary systems has been investigated in $[7-9,13,14]$. These works define the achievable transmission capacity as the spatial density of the successful transmissions per unit area, which neglects other system parameters. In [10], we investigate the achievable transmission capacity of cognitive networks in $\mathrm{bits} / \mathrm{hop} / \mathrm{s} / \mathrm{Hz} /$ node over different MAC schemes according to Shannon's theory and analyze how the network capacity is affected by the parameters of the secondary system rather than the spatial density. The numerical results in [10] indicate that secondary users can only access the spectrum at a low transmission power to preserve the outage probability constraints of both the primary and the secondary network over the underlay model.

Network relaying has been proposed in [15] as an approach to enhancing the total throughput and coverage of a wireless network. Its advantage lies in reducing the overall path loss by utilizing a relay between the source and the destination. Inspired by network relaying, cooperative cognitive relay networks (CCRN) have recently been investigated as a potential mechanism to improve the secondary network throughput. Two cooperation 
mechanisms have been proposed: cooperation among secondary users [16-19] and cooperation between primary and secondary users [20-25]. In the first case, secondary users access or sense the primary spectrum in a cooperative manner to enhance their performance; while in the second case, secondary users serve as relays for the primary network to achieve a 'win-win' situation, which is also the focus of this paper.

The cooperation between the primary and secondary systems under the interleave spectrum sharing model has been investigated in [20-22], which demonstrate that cooperation can support a higher stable performance for both the primary and the secondary users compared to non-cooperative networks. In [23], both the cooperation among SUs and that between PUs and SUs are simultaneously considered in a multi-hop cognitive radio network for multi-hop relaying. A trade-off on the utilities of the primary and the secondary users is studied in [24], and the results indicate that the primary and secondary users have the motivation to cooperate with each other if the performance of both systems can be dramatically improved when they cooperate. Kam et al.[25] investigate the queue stability of a two-user cooperative cognitive radio system with multicast traffic and demonstrate that the stability region of this cooperative approach is larger than that of the noncooperative approach, which benefits both users of this multicast system. Instead of studying the throughput, utility, or queue stability of a cognitive network over an interweave or overlay model, this paper intends to investigate the achievable transmission capacity of cooperative cognitive radio networks over an underlay model while preserving the outage probability constraints of both the primary and the secondary system.

Cooperative relaying has also been investigated in other network scenarios such as wireless LANs [26], sensor networks [27], and wideband sensory relay networks [28] to enhance the networking performance; these approaches are not applicable in an underlay cooperative cognitive radio networks as the relays do not simultaneously share the channel with the facilitated nodes. The capacity of general ad hoc networks has been extensively studied in literature $[29,30]$. The scaling laws of the throughput and/or delay of coexisting primary and secondary ad hoc networks under various protocol settings have been investigated in [29,31-33]. These works consider fundamentally different problems than our study, which focuses on the achievable transmission capacity (link layer) of cognitive radio networks with cooperative relaying.

\section{System model}

\subsection{Network model}

We consider a system model depicted in Figure 1, where a primary (licensed) transmitter PT communicates with an intended primary receiver PR. In the same spectrum band, a secondary network, composed of $N$ nodes, resides in the range of the primary network and is seeking to exploit possible transmission opportunities. When the PT is far from the PR, a secondary user, which has a better link quality and is not in transmitting or receiving mode, can be selected to relay packets for the PT. Such a scenario happens in a cooperative cognitive radio network, and the selected SU is called a cooperative relay. For simplicity, we assume that the primary user employs a fixed transmission power $P_{p}$, and all secondary transmitters have the same transmission power $P_{s}$.

We further assume that time is slotted, and that the transmission of one packet for both the primary and the secondary network takes the duration of exactly one time slot. If no cooperation is established between the PT and any SU, the PT transmits a packet to its PR per time slot while SUs are allowed to send their own packets at any slot as long as the accumulated interference experienced by the PR is tolerable. When a cooperative relay is employed, the delivery of a packet from the PT to the PR takes two time slots, with the first one for the transmission from the PT to the relay, and the second one from the relay to the PR. In other words, a packet from the PT takes one or two time slots depending on whether cooperative relaying is employed or not, while the SUs can transmit at any slot as long as their transmissions do not interfere with the reception at the PR.

\subsection{Physical layer model}

For a propagation channel model with a long-term path loss and a short-term independent flat Rayleigh fading, the received power at a typical receiver from a transmitter can be computed by $P_{k} \delta_{i j}\left|d_{i j}\right|^{-\alpha}$, where $P_{k}$ is the transmission power of network $k$, with $k=p$ denoting the primary network and $k=s$ denoting the secondary network, $\alpha$ is the path loss exponent, $d_{i j}$ is the distance between the transmitting node $i$ and the receiver $j$, and $\delta_{i j}$ is the fading factor on the power transmitted from the node $i$ to the receiver $j$. Let $i=0$ denote the PT, and $j=0$ denote the PR. Then, other values of $i$ and $j$ denote secondary users. In the Rayleigh fading channel, the probability density function of the fading factor $\delta_{i j}$ follows an exponential distribution with a unit mean $[7,9]$. Considering the cumulative interference from the transmitters of both the primary network and the secondary network, the SINR at the receiver $j$ of system $k$ can be represented by:

$$
\operatorname{SINR}_{i j}=\frac{P_{k} \delta_{i j} d_{i j}^{-\alpha}}{I_{p j}+I_{s j}+N_{0}},
$$

where $N_{0}$ is the thermal noise power, and $I_{p j}=$ $P_{p} \delta_{0 j}\left|d_{0 j}\right|^{-\alpha}$ is the cumulative interference power from the transmitting node of the primary network to the typical receiver $j$ of network $k$ and $I_{s j}=\sum_{q \in \mathrm{SU}} P_{s} \delta_{q j}\left|d_{q j}\right|^{-\alpha}$ (for 


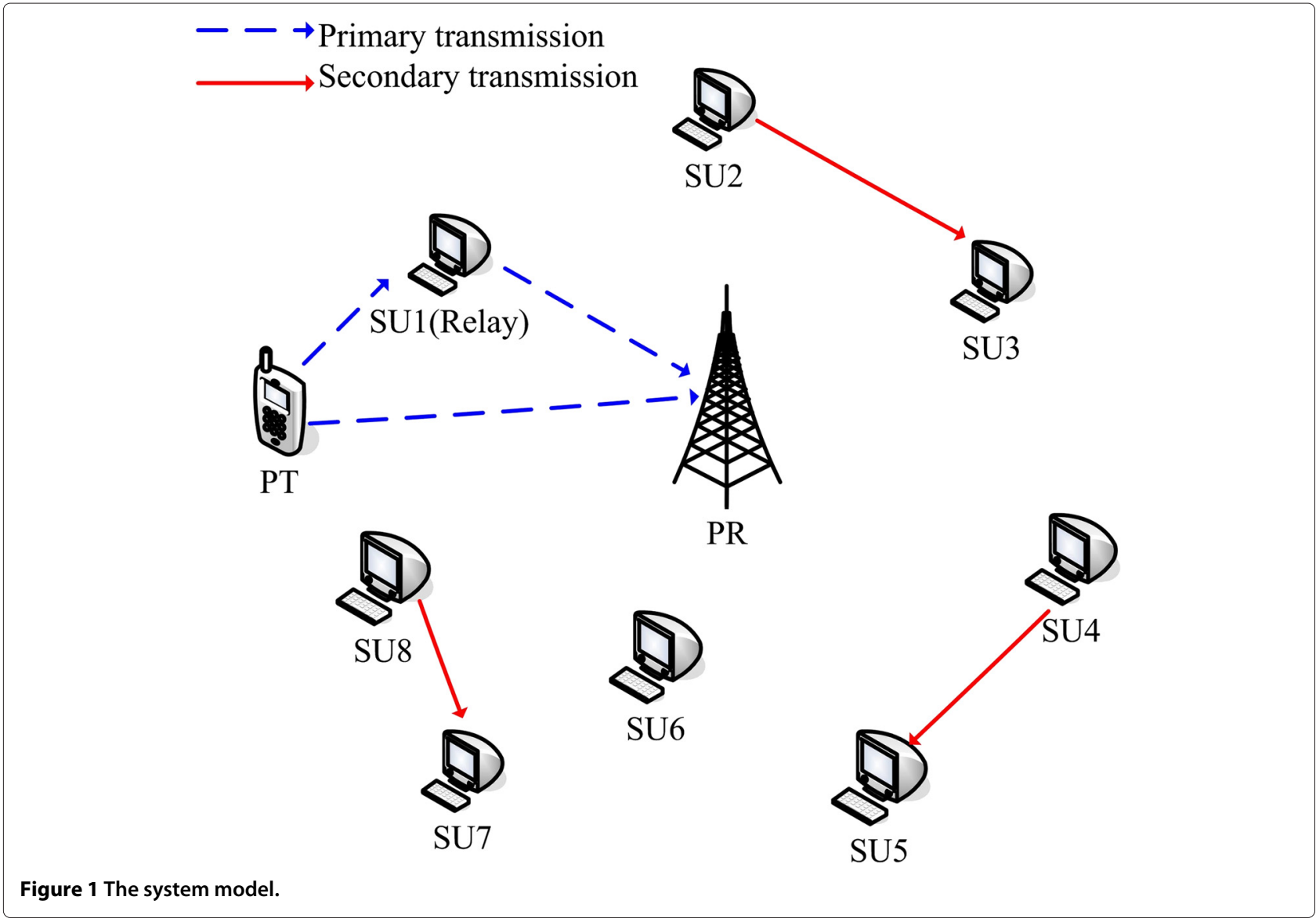

$q \neq i$ ) is the cumulative interference power from the transmitting node of the primary network to the typical receiver $j$ of network $k$. Note that $I_{p j}=0$ when $j=0$. As spectrum sharing systems are interference-limited [8], the thermal noise can be negligible. Hence, for simplicity, SIR is used instead of SINR:

$$
\operatorname{SIR}_{i j}=\frac{P_{k} \delta_{i j} d_{i j}^{-\alpha}}{I_{p j}+I_{s j}}
$$

A signal can be correctly decoded at a receiver of system $k$ if the corresponding SIR is greater than a threshold $\eta_{k}$. Thus, the probability of a successful transmission can be defined as $\operatorname{Pr}\left(S I R_{i j} \geq \eta_{k}\right)$.

\subsection{Achievable transmission capacity}

As claimed in [10], the achievable transmission capacity in packets/s/node does not take into account the spectral efficiency of each packet; thus we define the transmission capacity in bits/s/Hz/node, which measures the number of bits each node can receive from its desired transmitter per second per Hertz. A similar argument can be found in [34]. According to Shannon's theory, a packet can carry $\log _{2}\left(1+\eta_{s}\right)$ bits/s/Hz information. Thus, the achievable transmission capacity in bits $/ \mathrm{s} / \mathrm{Hz} /$ node of a secondary node can be defined as:

$$
C=\frac{1}{V} \log _{2}\left(1+\eta_{s}\right) \operatorname{Pr}\left(\operatorname{SIR}_{i j} \geq \eta_{s}\right)
$$

where $V$ is the number of time slots needed for each node in the network to transmit once to its neighbor. Particularly, in this paper, $V=1$ according to our previous assumption.

\section{Achievable transmission capacity of the secondary network without cooperative relaying}

As a baseline, we first analyze the achievable transmission capacity of the secondary network when no cooperative relay is utilized by the primary network. In such a case, the PT transmits signals to its PR directly. Assume that a subset of SUs, denoted by Sub, are allowed to transmit over the same spectrum band as the PT in each time slot, as long as their transmissions do not disturb the normal communications of the primary network. 
According to Equation 2, the successful transmission probability of the PT in each slot can be given as:

$$
\begin{aligned}
\operatorname{Pr}\left(\operatorname{SIR}_{00} \geq \eta_{p}\right) & =\operatorname{Pr}\left(\frac{P_{p} \delta_{00} d_{00}^{-\alpha}}{I_{s 0}} \geq \eta_{p}\right) \\
& =\operatorname{Pr}\left\{\delta_{00} \geq \frac{\eta_{p} d_{00}^{\alpha}}{P_{p}} I_{s 0}\right\} \\
& =\mathrm{E}_{\left\{\delta_{i 0}\right\}}\left\{\exp \left(-\frac{\eta_{p} d_{00}^{\alpha}}{P_{p}} \sum_{i \in\{\text { Sub }\}} P_{s} \delta_{i 0} d_{i 0}^{-\alpha}\right)\right\} \\
& =\prod_{i \in\{\text { Sub }\}} \frac{1+\frac{\eta_{p}}{\gamma_{p s}}\left(\frac{d_{00}}{d_{i 0}}\right)^{\alpha}}{}
\end{aligned}
$$

with $\gamma_{p s}=\frac{P_{p}}{P_{s}}$ being the power ratio between the primary and the secondary network.

Similarly, the successful transmission probability of a secondary user in each time slot is given by:

$$
\begin{aligned}
\operatorname{Pr}\left(\operatorname{SIR}_{i j} \geq \eta_{s}\right)= & \operatorname{Pr}\left(\frac{P_{s} \delta_{i j} d_{i j}^{-\alpha}}{I_{s j}+I_{p j}} \geq \eta_{s}\right) \\
= & \mathrm{E}_{\left\{\delta_{q j}\right\}}\left\{\exp \left(-\frac{\eta_{s} d_{i j}^{\alpha}}{P_{s}} \sum_{q \in\{S u b \backslash i\}} P_{s} \delta_{q j} d_{q j}^{-\alpha}\right)\right\} \\
& \times \mathrm{E}_{\left\{\delta_{0 j}\right\}} \exp \left(-\frac{\eta_{s} d_{i j}^{\alpha}}{P_{s}} P_{p} d_{0 j}^{-\alpha} \delta_{0 j}\right) \\
= & \prod_{q \in\{\operatorname{Sub} \backslash i\}} \frac{1+\eta_{s}\left(\frac{d_{i j}}{d_{q j}}\right)^{\alpha}}{1+\eta_{s} \gamma_{p s}\left(\frac{d_{i j}}{d_{0 j}}\right)^{\alpha}}
\end{aligned}
$$

Based on Equation 3, the achievable transmission capacity of the secondary user in each time slot can be computed as:

$$
\begin{aligned}
C= & \frac{1}{V} \log \left(1+\eta_{s}\right) \prod_{q \in\{\operatorname{Sub} \backslash i\}} \frac{1}{1+\eta_{s}\left(\frac{d_{i j}}{d_{q j}}\right)^{\alpha}} \\
& \times \frac{1}{1+\eta_{s} \gamma_{p s}\left(\frac{d_{i j}}{d_{0 j}}\right)^{\alpha}},
\end{aligned}
$$

which subjects to the following outage probability constraints:

$$
1-\operatorname{Pr}\left(\operatorname{SIR}_{00} \geq \eta_{p}\right) \leq \theta_{p}
$$

and:

$$
1-\operatorname{Pr}\left(\operatorname{SIR}_{i j} \geq \eta_{s}\right) \leq \theta_{s}
$$

where $\theta_{p}$ and $\theta_{s}$ are the maximum allowable outage probabilities of the primary and the secondary network, respectively.

Note that a bigger power ratio $\gamma_{p s}$ means a lower allowable transmission power while a smaller $\gamma_{p s}$ indicates a higher allowable transmission power for the SUs. In order to investigate the range of the allowable power ratio, we present an analysis on the first order derivative with respect to $\gamma_{p s}$. Let $f_{1}=1-\prod_{i \in\{\text { Sub }\}} \frac{1}{1+\frac{\eta_{p}}{\gamma_{p s}}\left(\frac{d_{00}}{d_{i 0}}\right)^{\alpha}}-\theta_{p}, a_{i}=$ $\eta_{p}\left(\frac{d_{00}}{d_{i 0}}\right)^{\alpha}$; then we have,

$$
\frac{\partial f_{1}}{\partial \gamma_{p s}}=-\sum_{i \in\{\operatorname{Sub}\}} \frac{a_{i}}{\left(1+a_{i} / \gamma_{p s}\right)^{2} \gamma_{p s}^{2}} \prod_{q \in\{\operatorname{Sub} \backslash i\}} \frac{1}{1+a_{q} / \gamma_{p s}}
$$

From Equation 9, we observe that $\frac{\partial f_{1}}{\partial \gamma_{p s}} \leq 0$. Thus, the value of $f_{1}$ decreases with the increase of $\gamma_{p s}$ when the other parameters are fixed. On the other hand, due to the requirement of Equation 7, i.e., the outage probability constraint of the primary network, $f_{1} \leq 0$. Hence, we can obtain the lower bound of the power ratio $\gamma_{p s}^{l}$ when $f_{1}=0$.

$$
\begin{aligned}
& \text { Also, let } f_{2}=1-\prod_{q \in\{\operatorname{Sub} \backslash i\}} \frac{1}{1+\eta_{s}\left(\frac{d_{i j}}{d_{q j}}\right)^{\alpha}} \frac{1}{1+\eta_{s} \gamma_{p s}\left(\frac{d_{i j}}{d_{0 j}}\right)^{\alpha}}-\theta_{s}, \\
& m=\eta_{s}\left(\frac{d_{i j}}{d_{0 j}}\right)^{\alpha} \text {, and } b=\prod_{q \in\{\operatorname{Sub} \backslash i\}} \frac{1}{1+\eta_{s}\left(\frac{d_{i j}}{d_{q j}}\right)^{\alpha}} \text {; then we have: } \\
& \frac{\partial f_{2}}{\partial \gamma_{p s}}=\frac{m b}{\left(1+m \gamma_{p s}\right)^{2}} .
\end{aligned}
$$

Since $\frac{\partial f_{2}}{\partial \gamma_{p s}} \geq 0$, the value of $f_{2}$ increases with the increase of $\gamma_{p s}$ when the other parameters are fixed. Moreover, to satisfy the outage probability constraint in Equation 8 of the secondary network, $f_{2} \leq 0$. Therefore, we can get the upper bound of the power ratio $\gamma_{p s}^{u}$ when $f_{2}=0$.

Furthermore, based on the observation that the SU's capacity decreases when the power ratio $\gamma_{p s}$ increases (see Equation 6), we can estimate the maximum achievable transmission capacity by substituting $\gamma_{p s}^{l}$ into Equation 6, i.e.,

$$
C^{*}=\frac{1}{V} \log \left(1+\eta_{s}\right) \prod_{q \in\{\text { Sub } \backslash i\}} \frac{1}{1+\eta_{s}\left(\frac{d_{i j}}{d_{q j}}\right)^{\alpha}} \frac{1}{1+\eta_{s} \gamma_{p s}^{l}\left(\frac{d_{i j}}{d_{0 j}}\right)^{\alpha}} .
$$

\section{Achievable transmission capacity of the secondary network with cooperative relaying}

In cooperative cognitive networks, a primary transmitter that is far away from its receiver can select a secondary user to relay its information. Two relaying protocols: DF and AF, can be adopted by the relay. For the DF protocol, the relay that successfully decodes the received message from the transmitter re-encodes the message and then transmits it to the destination. DF can be switched to the non-cooperative mode in the case of a failed cyclic redundancy check $(\mathrm{CRC})$ that may be resulted from the decoding errors. For the AF protocol, the relay simply amplifies the signal it receives. The destination combines two copies of the signal, with one directly from the source 
and the other via the relay, through a matched filter. Compared to DF, AF has a lower implementation complexity in digital signal processing at the relay node, and it can operate under all source-relay channel conditions; but it amplifies the noise power too when amplifying the useful signal [35].

Assume that the distance from the PT to the relay is $d_{0 r}$, and the distance from the relay to the PR is $d_{r 0}$. Notice that, in cooperative transmissions, the quality of the relayed link should be higher than that of the direct link, which implies that:

$$
\min \left\{\frac{P_{p} \delta_{0 r} d_{0 r}^{-\alpha}}{I_{s r}}, \frac{P_{s} \delta_{r 0} d_{r 0}^{-\alpha}}{I_{s 0}}\right\}>\frac{P_{p} \delta_{00} d_{00}^{-\alpha}}{I_{s 0}} .
$$

Therefore, we have:

$$
\left\{\begin{array}{l}
\frac{d_{0 r}^{-\alpha}}{I_{s r}}>\frac{d_{00}^{-\alpha}}{I_{s 0}} \\
d_{r 0}^{-\alpha}>\gamma_{p s} d_{00}^{-\alpha} .
\end{array}\right.
$$

Then, the location region of the feasible relays, in which the relays can help enhance the PU's transmission capacity, can be calculated as follows:

$$
\left\{\begin{aligned}
d_{0 r} & <\left(\frac{I_{s 0}}{I_{s r}}\right)^{\frac{1}{\alpha}} d_{00} \\
d_{r 0} & <\gamma_{p s}^{-\frac{1}{\alpha}} d_{00}
\end{aligned}\right.
$$

This indicates that the location region of the feasible relays is affected by the interference from the secondary users, the distance from the PT to the PR, as well as the power ratio. The above two equations in Equation 14 can be illustrated via Figure 2 . When the distance $d_{00}$ between the PT and the PR is fixed, the location region of the feasible relays, which is the shaded overlapping area of the two circles, is determined by the interference ratio $\frac{I_{s 0} 0}{I_{s r}}$ and the power ratio $\gamma_{p s}$. In other words, the relay selection depends on both the interference from other SUs and the transmit powers, i.e, $P_{p}$ and $P_{s}$. Moreover, the link quality and the PU's capacity can not be improved if the selected relay is out of the shaded overlapping area.

In the following two subsections, we investigate the achievable transmission capacity of a cognitive radio network with cooperative relaying when the relay respectively adopts the DF and the AF protocol.

\subsection{Decode-and-forward}

In the first time slot, the PT transmits a packet. According to our system model presented in Section 3, the successful receiving probability of the relay can be computed by:

$$
\begin{aligned}
\operatorname{Pr}\left(\operatorname{SIR}_{0 r} \geq \eta_{s}\right) & =\operatorname{Pr}\left(\frac{P_{p} \delta_{0 r} d_{0 r}^{-\alpha}}{I_{s r}} \geq \eta_{s}\right) \\
& =\operatorname{Pr}\left\{\delta_{0 r} \geq \frac{\eta_{s} d_{0 r}^{\alpha}}{P_{p}} I_{s r}\right\} \\
& =\prod_{i \in\{\text { Sub }\}} \frac{1}{1+\frac{\eta_{s}}{\gamma_{p s}}\left(\frac{d_{0 r}}{d_{i r}}\right)^{\alpha}} .
\end{aligned}
$$

For simplicity, we denote the first time slot by the superscript ' 1 ' and the second one by the superscript ' 2 '. The successful transmission probability of a secondary user in the first slot is the same as that of the case without cooperative relaying, i.e.,

$$
\operatorname{Pr}_{\mathrm{DF}}^{1}\left(\operatorname{SIR}_{i j} \geq \eta_{s}\right)=\prod_{q \in\{\operatorname{Sub} \backslash i\}} \frac{1}{1+\eta_{s}\left(\frac{d_{i j}}{d_{q j}}\right)^{\alpha}} \frac{1}{1+\eta_{s} \gamma_{p s}\left(\frac{d_{i j}}{d_{0 j}}\right)^{\alpha}}
$$

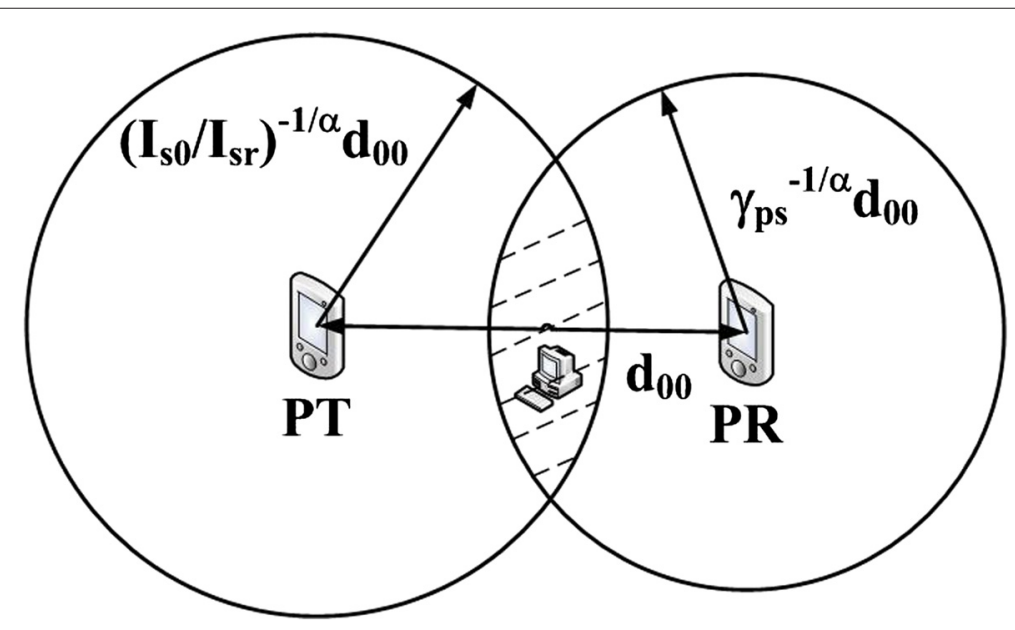

Figure 2 The location region of the feasible relays. 
Thus, the achievable transmission capacity of a secondary user is:

$$
\begin{aligned}
C_{\mathrm{DF}}^{1}= & \frac{1}{V} \log \left(1+\eta_{s}\right) \prod_{q \in\{\operatorname{Sub} \backslash i\}} \frac{1}{1+\eta_{s}\left(\frac{d_{i j}}{d_{q j}}\right)^{\alpha}} \\
& \times \frac{1}{1+\eta_{s} \gamma_{p s}\left(\frac{d_{i j}}{d_{0 j}}\right)^{\alpha}} .
\end{aligned}
$$

In the second slot, the relay re-encodes the message received from the PT and transmits it to the PR. Hence, the successful transmission probability from the relay to the PR is:

$$
\begin{aligned}
\operatorname{Pr}\left(\operatorname{SIR}_{r 0} \geq \eta_{p}\right) & =\operatorname{Pr}\left(\frac{P_{s} \delta_{r 0} d_{r 0}^{-\alpha}+P_{p} \delta_{00} d_{00}^{-\alpha}}{I_{s 0}} \geq \eta_{p}\right) \\
& =\prod_{i \in\{\operatorname{Sub}\}} \frac{1}{1+\eta_{p}\left(\frac{d_{r 0}}{d_{i 0}}\right)^{\alpha}} \frac{1}{1-\gamma_{p s}\left(\frac{d_{r 0}}{d 00}\right)^{\alpha}} .
\end{aligned}
$$

The successful transmission probability for a secondary user $i$ to reach its (secondary) destination $j$ is:

$$
\begin{aligned}
\operatorname{Pr}_{\mathrm{DF}}^{2}\left(\operatorname{SIR}_{i j} \geq \eta_{s}\right) & =\operatorname{Pr}\left(\frac{P_{s} \delta_{i j} d_{i j}^{-\alpha}}{I_{s j}+I_{r j}} \geq \eta_{s}\right) \\
& =\prod_{q \in\{\mathrm{Sub} \backslash i\}} \frac{1}{1+\eta_{s}\left(\frac{d_{i j}}{d_{q j}}\right)^{\alpha}} \frac{1}{1+\eta_{s}\left(\frac{d_{i j}}{d_{r j}}\right)^{\alpha}} .
\end{aligned}
$$

Then, the achievable transmission capacity of a secondary user can be given by:

$$
C_{\mathrm{DF}}^{2}=\frac{1}{V} \log \left(1+\eta_{s}\right) \prod_{q \in\{\mathrm{Sub} \backslash i\}} \frac{1}{1+\eta_{s}\left(\frac{d_{i j}}{d_{q j}}\right)^{\alpha}} \frac{1}{1+\eta_{s}\left(\frac{d_{i j}}{d_{r j}}\right)^{\alpha}} .
$$

The outage probability for the transmission from PT to $\mathrm{PR}$ is $1-\operatorname{Pr}\left(\operatorname{SIR}_{0 r} \geq \eta_{s}\right) \operatorname{Pr}\left(\operatorname{SIR}_{r 0} \geq \eta_{p}\right)$. Hence, from Equations 15 20, the average transmission capacity of a secondary user with outage probability constraints from both the primary and the secondary network can be derived as follows:

$$
\begin{aligned}
C_{\mathrm{DF}}= & \frac{1}{2 V} \log \left(1+\eta_{s}\right) \prod_{q \in\{\mathrm{Sub} \backslash i\}} \frac{1}{1+\eta_{s}\left(\frac{d_{i j}}{d_{q j}}\right)^{\alpha}}\left[\frac{1}{1+\eta_{s} \gamma_{p s}\left(\frac{d_{i j}}{d_{0 j}}\right)^{\alpha}}\right. \\
& \left.+\frac{1}{1+\eta_{s}\left(\frac{d_{i j}}{d_{r j}}\right)^{\alpha}}\right]
\end{aligned}
$$

subject to the following outage probability constraints:

$$
\begin{aligned}
& 1-\operatorname{Pr}\left(\operatorname{SIR}_{0 r} \geq \eta_{s}\right) \operatorname{Pr}\left(\operatorname{SIR}_{r 0} \geq \eta_{p}\right) \leq \theta_{p}, \\
& 1-\operatorname{Pr}_{\mathrm{DF}}^{1}\left(\operatorname{SIR}_{i j} \geq \eta_{s}\right) \leq \theta_{s}
\end{aligned}
$$

and:

$$
1-\operatorname{Pr}_{\mathrm{DF}}^{2}\left(\operatorname{SIR}_{i j} \geq \eta_{s}\right) \leq \theta_{s}
$$

Similar to the analysis in Section 4, from Equations 22 and 23, we obtain respectively the lower bound $\gamma_{p s}^{l}$ and the upper bound $\gamma_{p s}^{u}$ of the power ratio, which are affected by the location of the relay. In addition, from Equation 21, we observe that the capacity of the secondary user decreases with the increase of the power ratio. Thus, by substituting $\gamma_{p s}^{l}$ into Equation 21, we obtain the following maximum transmission capacity $C_{\mathrm{DF}}^{*}$ of the cooperative cognitive network:

$$
\begin{aligned}
C_{\mathrm{DF}}^{*}= & \frac{1}{2 V} \log \left(1+\eta_{s}\right) \prod_{q \in\{\mathrm{Sub} \backslash i\}} \frac{1}{1+\eta_{s}\left(\frac{d_{i j}}{d_{q j}}\right)^{\alpha}}\left[\frac{1}{1+\eta_{s} \gamma_{p s}^{l}\left(\frac{d_{i j}}{d_{0 j}}\right)^{\alpha}}\right. \\
& \left.+\frac{1}{1+\eta_{s}\left(\frac{d_{i j}}{d_{r j}}\right)^{\alpha}}\right] .
\end{aligned}
$$

\subsection{Amplify-and-forward}

The transmission scheme of the AF protocol is the same as that of the DF protocol in the first slot. Hence, the successful transmission probability and the achievable transmission capacity of a secondary user has the same expression as that in the DF protocol; that is,

$$
\begin{aligned}
\operatorname{Pr}_{\mathrm{AF}}^{1}\left(\operatorname{SIR}_{i j} \geq \eta_{s}\right)= & \prod_{q \in\{\operatorname{Sub} \backslash i\}} \frac{1}{1+\eta_{s}\left(\frac{d_{i j}}{d_{q j}}\right)^{\alpha}} \\
& \times \frac{1}{1+\eta_{s} \gamma_{p s}\left(\frac{d_{i j}}{d_{0 j}}\right)^{\alpha}},
\end{aligned}
$$

and:

$$
\begin{aligned}
C_{\mathrm{AF}}^{1}= & \frac{1}{V} \log \left(1+\eta_{s}\right) \prod_{q \in\{\operatorname{Sub} \backslash i\}} \frac{1}{1+\eta_{s}\left(\frac{d_{i j}}{d_{q j}}\right)^{\alpha}} \\
& \times \frac{1}{1+\eta_{s} \gamma_{p s}\left(\frac{d_{i j}}{d_{0 j}}\right)^{\alpha}} .
\end{aligned}
$$

The received signal power at the relay can be written as:

$$
P_{r}=P_{0 r}+\sum_{i \in \mathrm{SU}} P_{i r}
$$

where $P_{0 r}=P_{p} d_{0 r}^{-\alpha} \delta_{0 r}$ is the signal power received from PT, $P_{i r}=P_{s} d_{i r}^{-\alpha} \delta_{i r}(i \neq 0)$ is the signal power received from the secondary transmitter $i$. Note that $P_{0 r}$ and $P_{i r}$ are random variables obeying exponential distributions with expectations $\mu_{0}=P_{p} d_{0 r}^{-\alpha}$ and $\mu_{i}=P_{s} d_{i r}^{-\alpha}$, respectively [36]. 
After the transmission in the first slot is over, the relay amplifies the received signal power to $P_{S}$ and then forwards it to the destination. Define $\beta_{1}=\frac{\mu_{0}}{\mu_{0}+\sum_{i \in S \mathrm{~S}} \mu_{i}}$ and $\beta_{2}=\frac{\sum_{i \in \mathrm{SU}} \mu_{i}}{\mu_{0}+\sum_{i \in \mathrm{SU}} \mu_{i}}$. Then, the successful decoding probability of the signal from the relay at the destination can be given by:

$$
\begin{aligned}
\operatorname{Pr}\left(\operatorname{SIR}_{r 0} \geq \eta_{p}\right)= & \operatorname{Pr}\left(\frac{\beta_{1} P_{s} \delta_{r 0} d_{r 0}^{-\alpha}+P_{p} \delta_{00} d_{00}^{-\alpha}}{\beta_{2} P_{s} \delta_{r 0} d_{r 0}^{-\alpha}+I_{s 0}} \geq \eta_{p}\right) \\
= & \operatorname{Pr}\left(\delta_{r 0} \geq \frac{\eta_{p} \beta_{2}}{\beta_{1}}+\sum_{i \in S \mathrm{SU}} \frac{\eta_{p} d_{r 0}^{\alpha}}{\beta_{1} d_{i 0}^{\alpha}} \delta_{i 0}\right. \\
& \left.-\frac{P_{p} d_{r 0}^{\alpha}}{\beta_{1} P_{s} d_{00}^{\alpha}} \delta_{00}\right) \\
= & \frac{1}{1+\eta_{p} \frac{\beta_{2}}{\beta_{1}}} \prod_{i \in \mathrm{SU}} \frac{1}{1+\frac{\eta_{p}}{\beta_{1}}\left(\frac{d_{r 0}}{d_{i 0}}\right)^{\alpha}} \\
& \times \frac{1}{1-\frac{\gamma_{p s}}{\beta_{1}}\left(\frac{d_{r 0}}{d_{00}}\right)^{\alpha} .}
\end{aligned}
$$

The successful transmission probability and the achievable transmission capacity for a secondary user at the secondary slot can be expressed as:

$$
\operatorname{Pr}_{\mathrm{AF}}^{2}\left(\operatorname{SIR}_{i j} \geq \eta_{s}\right)=\prod_{q \in\{\mathrm{Sub} \backslash i\}} \frac{1}{1+\eta_{s}\left(\frac{d_{i j}}{d_{q j}}\right)^{\alpha}} \frac{1}{1+\eta_{s}\left(\frac{d_{i j}}{d_{r j}}\right)^{\alpha}},
$$

and:

$$
C_{\mathrm{AF}}^{2}=\frac{1}{V} \log \left(1+\eta_{s}\right) \prod_{q \in\{\mathrm{Sub} \backslash i\}} \frac{1}{1+\eta_{s}\left(\frac{d_{i j}}{d_{q j}}\right)^{\alpha}} \frac{1}{1+\eta_{s}\left(\frac{d_{i j}}{d_{r j}}\right)^{\alpha}} .
$$

Then, the average transmission capacity of a secondary user with outage probability constraints from both the primary and the secondary network can be given as follows:

$$
\begin{aligned}
C_{\mathrm{AF}} & =\frac{1}{2}\left(C_{\mathrm{AF}}^{1}+C_{\mathrm{AF}}^{2}\right) \\
& =\frac{1}{2 V} \log \left(1+\eta_{s}\right) \prod_{q \in\{\mathrm{Sub} \backslash i\}} \frac{1}{1+\eta_{s}\left(\frac{d_{i j}}{d_{q j}}\right)^{\alpha}}\left[\frac{1}{1+\eta_{s} \gamma_{p s}\left(\frac{d_{i j}}{d_{0 j}}\right)^{\alpha}}\right. \\
& \left.+\frac{1}{1+\eta_{s}\left(\frac{d_{i j}}{d_{r j}}\right)^{\alpha}}\right] .
\end{aligned}
$$

Correspondingly, the outage probability constraints are:

$$
1-\operatorname{Pr}\left(\operatorname{SIR}_{r 0} \geq \eta_{p}\right) \leq \theta_{p},
$$

$$
1-\operatorname{Pr}_{\mathrm{AF}}^{1}\left(\operatorname{SIR}_{i j} \geq \eta_{s}\right) \leq \theta_{s}
$$

and:

$$
1-\operatorname{Pr}_{\mathrm{AF}}^{2}\left(\operatorname{SIR}_{i j} \geq \eta_{s}\right) \leq \theta_{s} .
$$

From Equations 33 and 34, we can obtain the lower bound $\gamma_{p s}^{l}$ and the upper bound $\gamma_{p s}^{u}$ of the power ratio, respectively. Since the capacity of the secondary user decreases with the increase of the power ratio, we can obtain the following maximum transmission capacity $C_{\mathrm{AF}}^{*}$ of the cooperative cognitive network by substituting $\gamma_{p s}^{l}$ into Equation 32.

$$
\begin{aligned}
C_{\mathrm{AF}}^{*}= & \frac{1}{2 V} \log \left(1+\eta_{s}\right) \prod_{q \in\{\mathrm{Sub} \backslash i\}} \frac{1}{1+\eta_{s}\left(\frac{d_{i j}}{d_{q j}}\right)^{\alpha}}\left[\frac{1}{1+\eta_{s} \gamma_{p s}^{l}\left(\frac{d_{i j}}{d_{0 j}}\right)^{\alpha}}\right. \\
& \left.+\frac{1}{1+\eta_{s}\left(\frac{d_{i j}}{d_{r j}}\right)^{\alpha}}\right]
\end{aligned}
$$

\subsection{Summary}

Adopting cooperative communications aims to enhance the performance of both the primary and the secondary network, achieving a 'win-win' situation. Based on our previous derivation and analysis, we draw the following important conclusions.

- As illustrated by Equation 14, the PU's transmission capacity can be improved when the selected relay falls in the location region satisfying $d_{0 r}<\left(\frac{I_{s 0}}{I_{s r}}\right)^{\frac{1}{\alpha}} d_{00}$ and $d_{r 0}<\gamma_{p s}^{-\frac{1}{\alpha}} d_{00}$. In addition, the performance improvement may depend on the type of the relaying protocol (i.e., DF or AF).

- The impact on the lower bound of the power ratio $\gamma_{p s}^{l}$ comes from the decision whether or not to cooperate with the PUs, the selection of the relaying protocol, as well as the interference from the neighboring SUs (see Equations 7, 22, and 33).

- The upper bound of the power ratio $\gamma_{p s}^{u}$ is determined by the decision whether or not to cooperate with the PUs and the interference from neighboring SUs (see Equations 8, 23, 24, 34, and 35).

\section{Numerical analysis}

In this section, we report our numerical results on the achievable transmission capacity of the cognitive network with/without cooperative relaying. For simplicity, we consider a simple network shown in Figure 3, where one relay coexists with a PT-PR pair, two transmitters of the secondary network have the same distance to the PR, and the number of time slots needed for each secondary node to transmit once to its neighbor is set to 1 . For simplicity, we first consider the case when the relay resides in the PTPR line. The impact of the relay position will be studied 

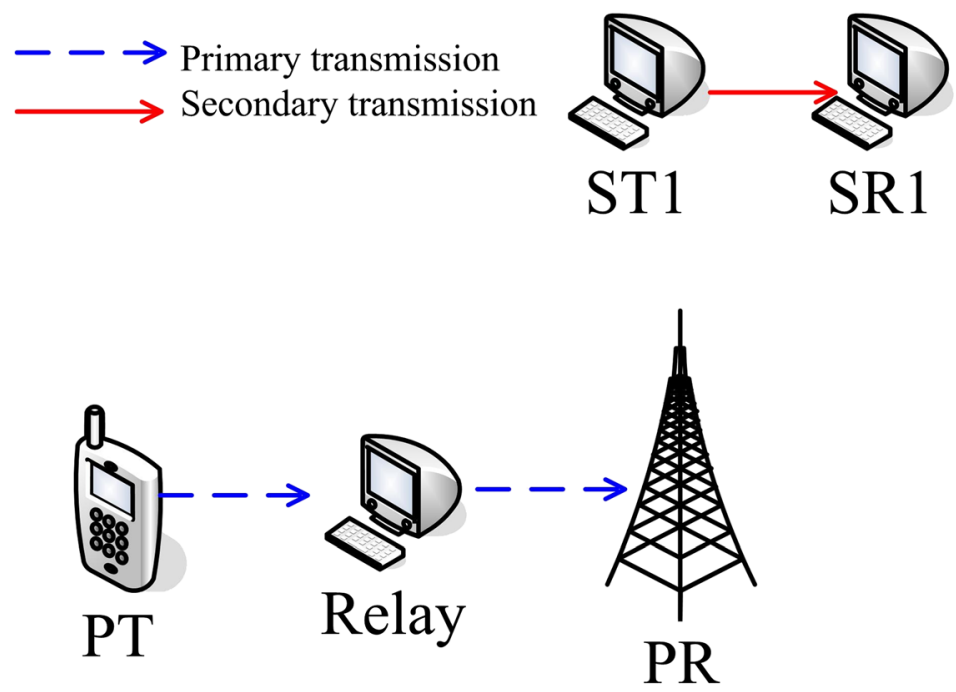

Figure 3 The topology utilized for the numerical analysis.

later. As elaborated in the following subsections, such a simple topology can perfectly capture the insights of our analysis while facilitating the thorough comprehension of the numerical results. The distance calculation as well as other parameter settings utilized in our simulation study are listed in Table 1. These settings ensure that the PR is located in the ST1-ST2 line and that the ST1-ST2 line is perpendicular to the PT-PR line.
Figure 4 reports the achievable transmission capacity versus the power ratio when the relay is 20,50 , or $80 \mathrm{~m}$ away from the PR in the PT-PR line. From the figure, we observe that there exists an upper bound and a lower bound for the power ratio to satisfy the outage probability constraints when other parameters are fixed for the cognitive network with cooperative relaying. For example, when the relay is fixed to be $20 \mathrm{~m}$ away from the PR, the network

Table 1 The parameter settings for the simulation study

\begin{tabular}{lll}
\hline Symbol & Semantic meaning & Value \\
\hline$\alpha$ & Pass loss exponent & 4 \\
$\eta_{p}$ & Threshold of the PR in the primary network & $4 \mathrm{~dB}$ \\
$d_{00}$ & Distance between PT and PR & $d$ \\
$d_{i 0}$ & Distance between a secondary transmitter and the PR & $100 \mathrm{~m}$ \\
$d_{i j}$ & Distance between a secondary transmitter and its destination & $20 \mathrm{~m}$ \\
$d_{0 j}$ & Distance between the PT and a secondary receiver & $\sqrt{120^{2}+100^{2}} \mathrm{~m}$ \\
$\beta$ & Angle between the line of Relay-PR and that of PT-PR & $0, \frac{\pi}{3}$ \\
$d_{r 0}$ & Distance between relay and PR & $d r$ \\
$d_{0 r}$ & Distance between PT and relay & $\sqrt{(d-d r \cdot \cos \beta)^{2}+(d r \cdot \sin \beta)^{2}} \mathrm{~m}$ \\
$d_{i r}$ & Distance between the secondary transmitter and the relay & $\sqrt{100^{2}+d^{2}} \mathrm{~m}$ \\
$d_{r j}$ & Distance between the relay and a secondary receiver & $\sqrt{100^{2}+(d+20)^{2}} \mathrm{~m}$ \\
\hline
\end{tabular}




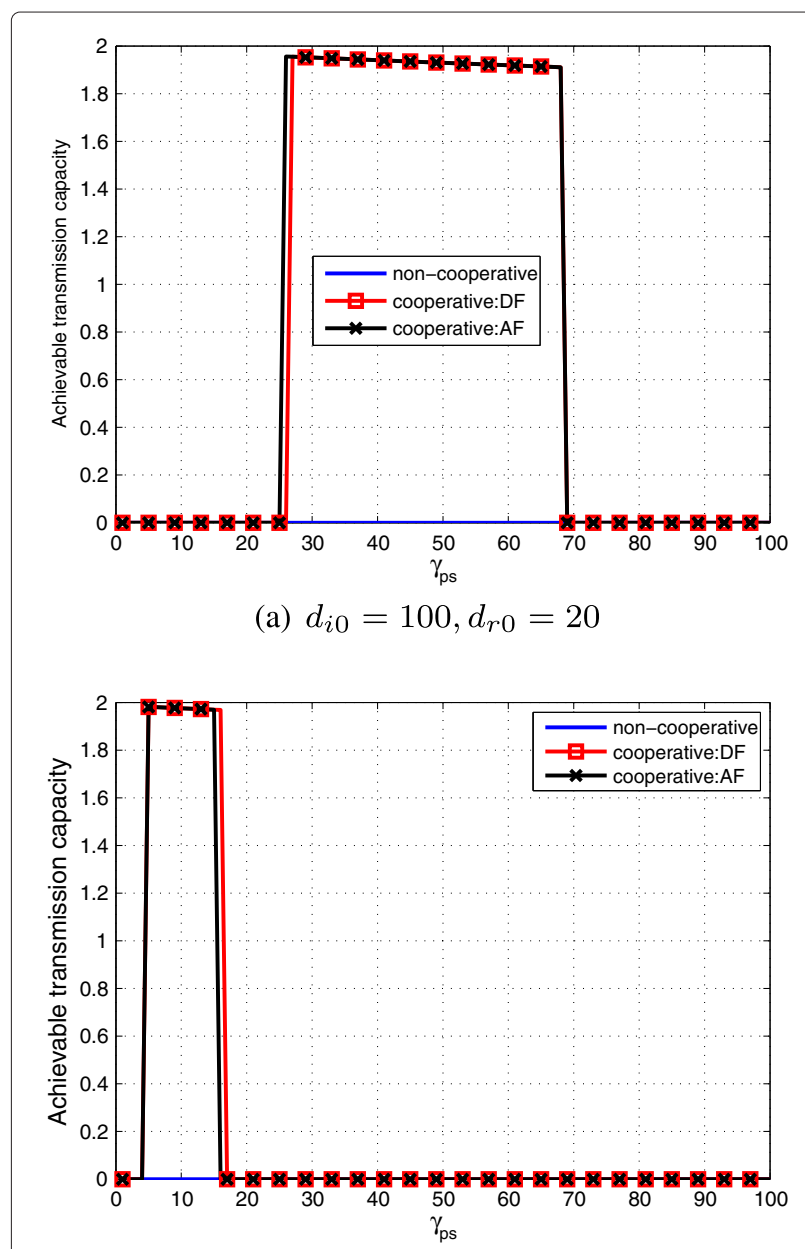

(b) $d_{i 0}=100, d_{r 0}=50$

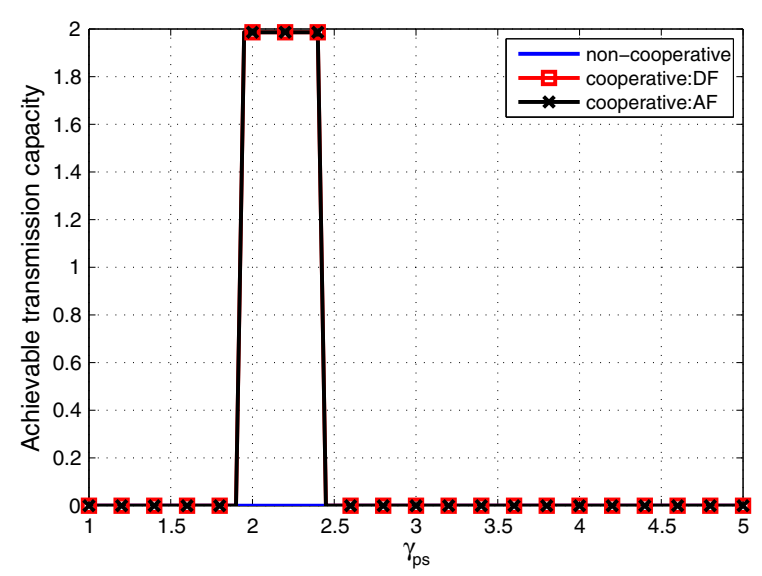

(c) $d_{i 0}=100, d_{r 0}=80$

Figure 4 Achievable transmission capacity of the cognitive network versus the power ratio when $d_{r 0}$ varies. (a) $d_{i 0}=100$, $d_{r 0}=20$, (b) $d_{i 0}=100, d_{r 0}=50$, and (c) $d_{i 0}=100, d_{r 0}=80$.

can achieve a certain capacity only when the power ratio is higher than 26 and lower than 69 . The feasible range of the power ratio becomes smaller when the distance from the relay to the PR becomes larger. Note that no transmission capacity can be achieved when the cognitive network does not provide cooperative relaying.

Since the transmission capacity decreases with the increase of the power ratio, we also investigate the optimal power ratio in this simulation study. Figure 5 reports the optimal power ratio versus the distance from the relay to the PR along the PT-PR line. From this figure, we observe that the optimal power ratio decreases with the increase of the distance from the relay to the PR. This is because when the relay is nearer to the PR, the PR experiences a lower pass loss such that the secondary user can increase its transmission power for capacity enhancement. The maximum achievable transmission capacity when adopting the optimal power ratio is given in Figure 6, in which the threshold of the secondary network is set to be 1 or 3 . This figure indicates that when the threshold equals 1 , the network can achieve a certain capacity with or without cooperative relaying but it achieves a higher capacity when cooperative relaying is provided to the primary network. Since the outage probability increases with the increase of the threshold and the power ratio, the outage probability constraints may not be guaranteed in the network without cooperation according to Equations 8, 23, 24, 34, and 35. When the threshold increases to 3 , the maximum transmission capacity for the network with cooperative relaying can be achieved only when the distance from the relay to the PR is higher than 10 where the outage probability constraints are satisfied. For the network without providing cooperative relaying, it achieves a capacity of 0 , as shown in Figure 6b.

Figure 7 reports the maximum achievable transmission capacity versus threshold when the relay is 20,50 , and $80 \mathrm{~m}$ away from the PR along the PT-PR line. From the subfigures in Figure 7, we notice that the maximum transmission capacities of both cases (with and without cooperative

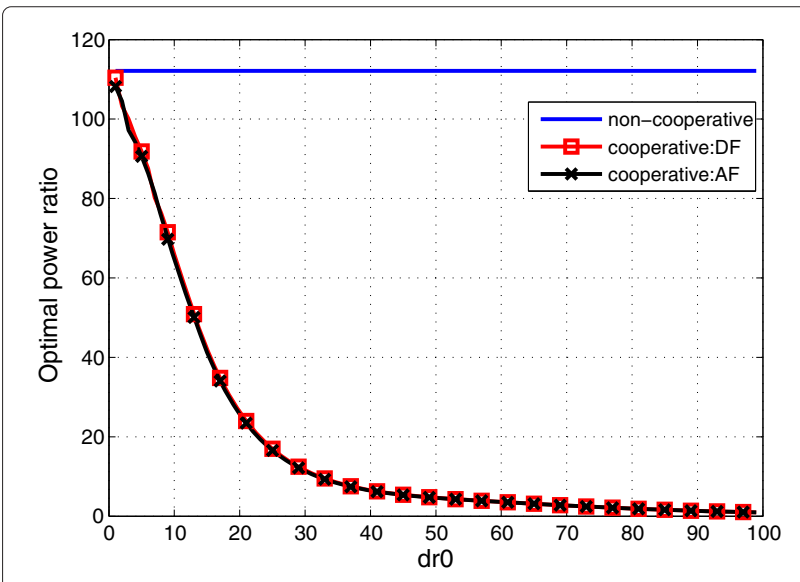

Figure 5 The optimal power ratio versus the distance from the relay to the primary receiver $\left(d_{i 0}=100\right)$. 


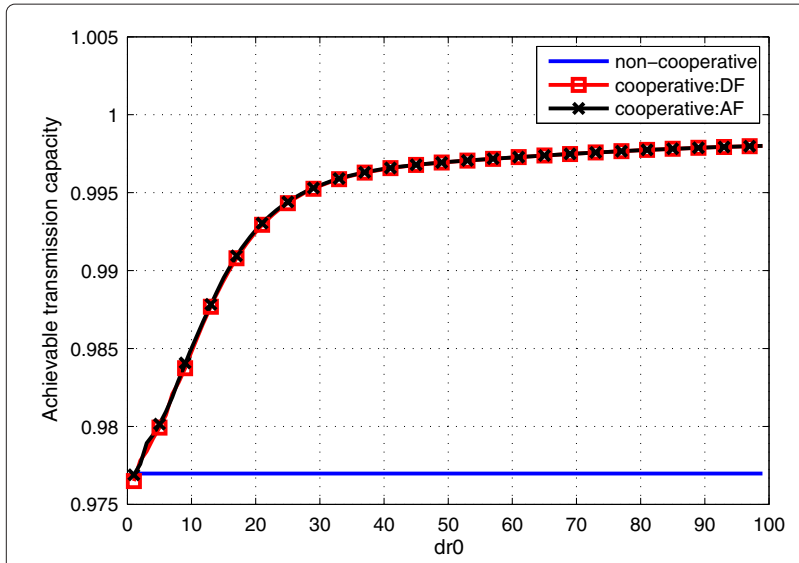

(a) threshold $\eta_{s}=1, d_{i 0}=100$

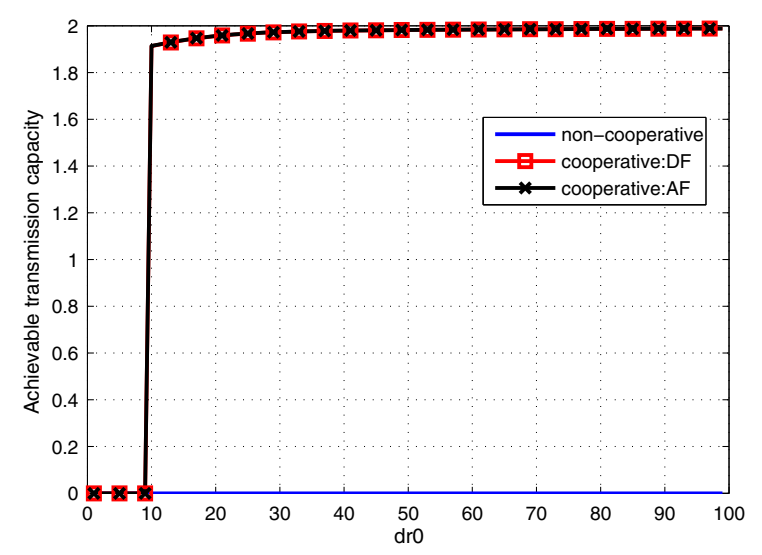

(b) threshold $\eta_{s}=3, d_{i 0}=100$

Figure 6 Maximum achievable transmission capacity of the cognitive network versus the distance from the relay to the primary receiver. (a) Threshold $\eta_{s}=1, d_{i 0}=100$ and (b) Threshold $\eta_{s}=3, d_{i 0}=100$.

relaying) increase with the increase of $\eta_{s}$ when $\eta_{s}$ is below a certain value and plummet to zero when $\eta_{s}$ exceeds a certain value. When the relay is $20 \mathrm{~m}$ away from the $\mathrm{PR}$, the maximum receiving threshold $\eta_{s}$ of the secondary network can not be higher than 2 for the cognitive network without providing cooperative relaying in order to preserve the outage probability constraints from both the primary and the secondary system. This threshold value reaches 5 and 6 with cooperative relaying over DF and AF, respectively. When the distance from the relay to the PR increases, the maximum threshold for cooperative relaying increases. For example, it reaches a value that is more than 30 when the relay is set to $80 \mathrm{~m}$ away from PR. This can be explained as follows: when the relay is nearer to the PR, the PR experiences a lower pass loss such that the secondary user can increase its transmission power. Accordingly, the secondary receiver can achieve a higher

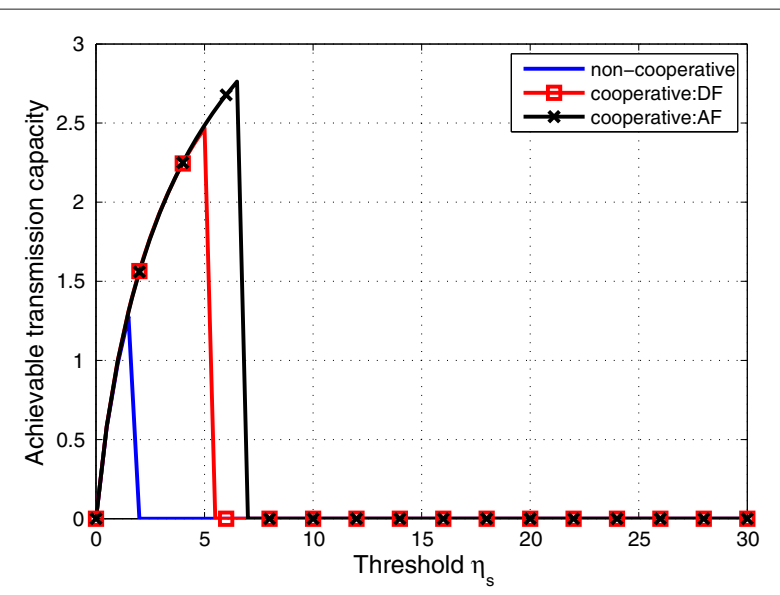

(a) $d_{i 0}=100, d_{r 0}=20$

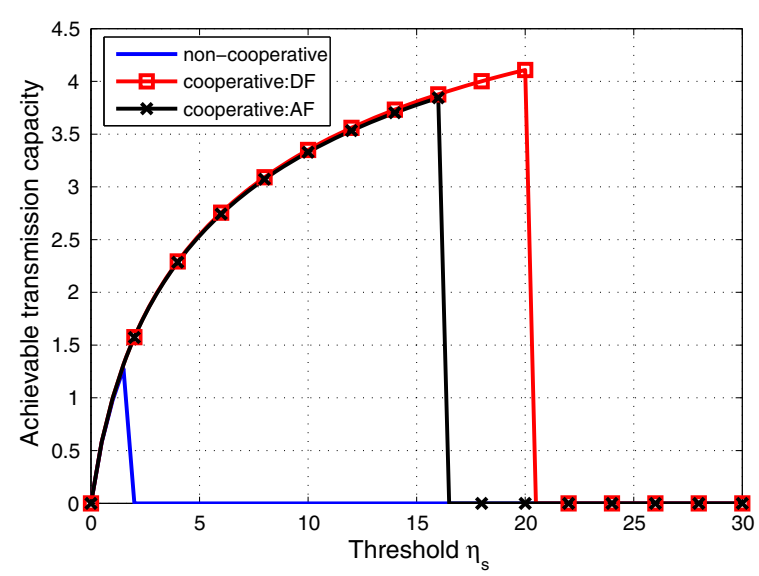

(b) $d_{i 0}=100, d_{r 0}=50$

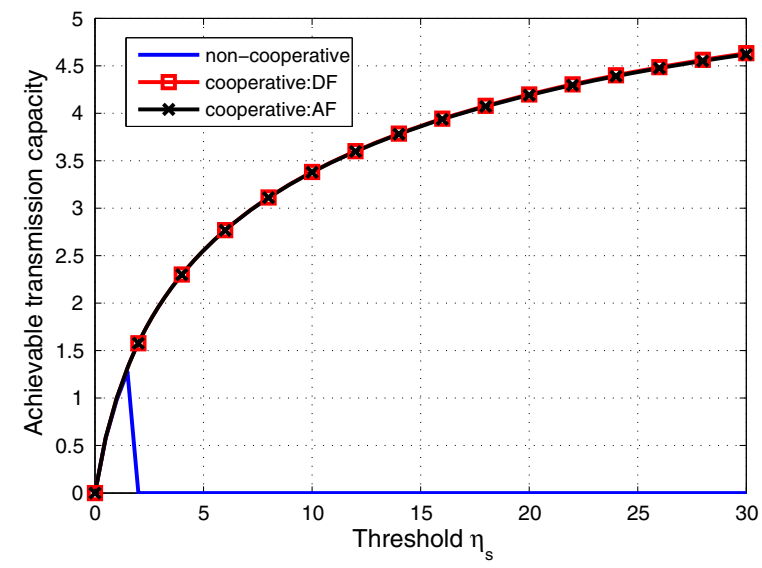

(c) $d_{i 0}=100, d_{r 0}=80$

Figure 7 The maximum achievable transmission capacity of the cognitive network when the location of the relay varies.

(a) $d_{i 0}=100, d_{r 0}=20$, (b) $d_{i 0}=100, d_{r 0}=50$, and (c) $d_{i 0}=100$, $d_{r 0}=80$.

SIR when the power ratio between the primary and the secondary network decreases. Thus, the secondary user can receive its signal successfully with a higher threshold. 
Given a higher threshold $\eta_{s}$, the network with cooperative relaying can achieve a larger capacity than the one without cooperative relaying.

Moreover, the results in Figure 7 also indicate that the AF protocol outperforms the DF protocol when the relay is $20 \mathrm{~m}$ away from the PR, but it yields a lower performance when the relay is located in the middle between the PT and the PR. This is due to the fact that when the relay is nearer to the PR, the loss caused by the amplified noise power when relaying the message based on the AF protocol is lower than that caused by the outage probability constraint from the primary user when decoding the message in the DF protocol, and this cost becomes a little higher when the relay located in the middle between the PT and the PR.

The numerical results of the maximum transmission capacities of the network with and without cooperative relaying versus the receiver threshold $\eta_{s}$ of the secondary network are reported in Figure 8 (a), (b), and (c) when the distance between the PT and the PR is set to 100 , 50 , and 20 , respectively. When the PT is $100 \mathrm{~m}$ away from the $\mathrm{PR}$, the maximum receiving threshold $\eta_{s}$ of the secondary network can not exceed 2 in the non-cooperative case, while it can increase to 20 for cooperative relaying over the DF protocol and to16 over the AF protocol in order to preserve the outage probability constraints from both the primary and the secondary network. The capacity can be improved by about $300 \%$ with an optimal threshold and power ratio in the cognitive network with cooperative relaying. When the distance of the PT is set to $50 \mathrm{~m}$ away from the PR, the capacity gain of cooperative relaying decreases though the network with or without cooperative relaying can achieve a higher capacity. When the distance between the PT and the PR decreases to $20 \mathrm{~m}$, the maximum threshold with cooperative relaying is about 27, while it is higher than 30 for non-cooperative relaying, and the cooperation between the PU and the SU makes an adverse effect on the maximum achievable transmission capacity of the cognitive network. This is due to the fact that the transmission between the PT and the PR experiences a lower pass loss when the PT is nearer to the PR, and the cost caused by the interference from the relay is higher than the advantage of reducing the pass loss via a cooperative relay.

Finally we investigate the impact of the relay location on the achievable transmission capacity. In our simulation, the change of the relay position is quantified by $\beta$, the angle between the relay-PR line and the PTPR line. This implies that our previous numerical study (Figures 4, 5, 6, 7 and 8) corresponds to the case of $\beta=0$. As indicated by (14) and Figure 2 in Section 5, the feasible location region of the relay is determined by the interferences from other SUs and the transmit powers; more specifically, it is determined by the interference ratio

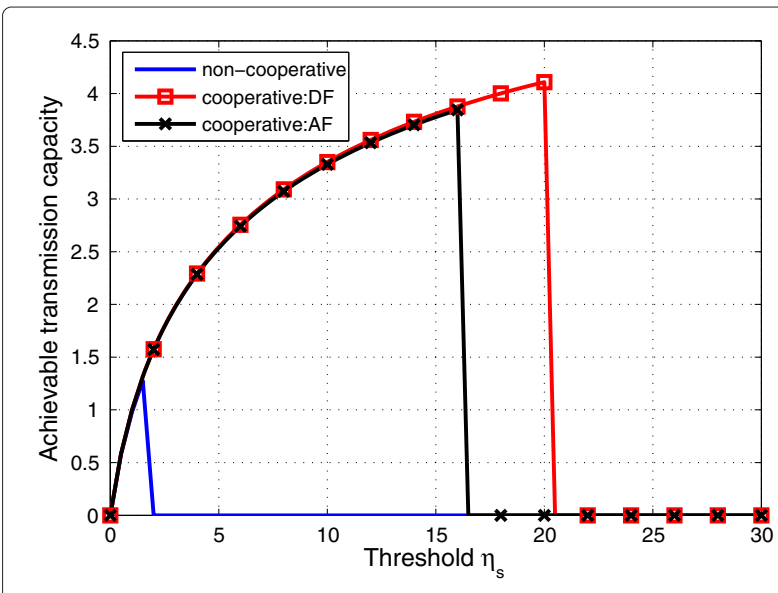

(a) $d_{i 0}=100, d_{00}=100$

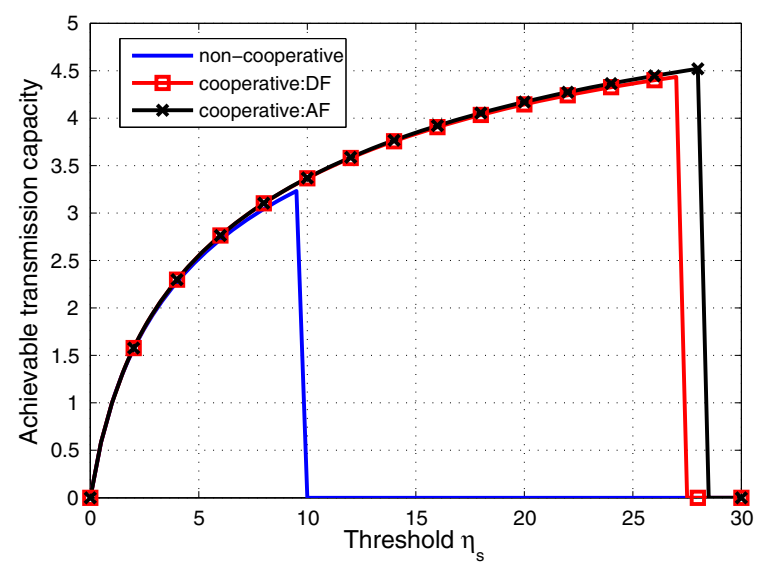

(b) $d_{i 0}=100, d_{00}=50$

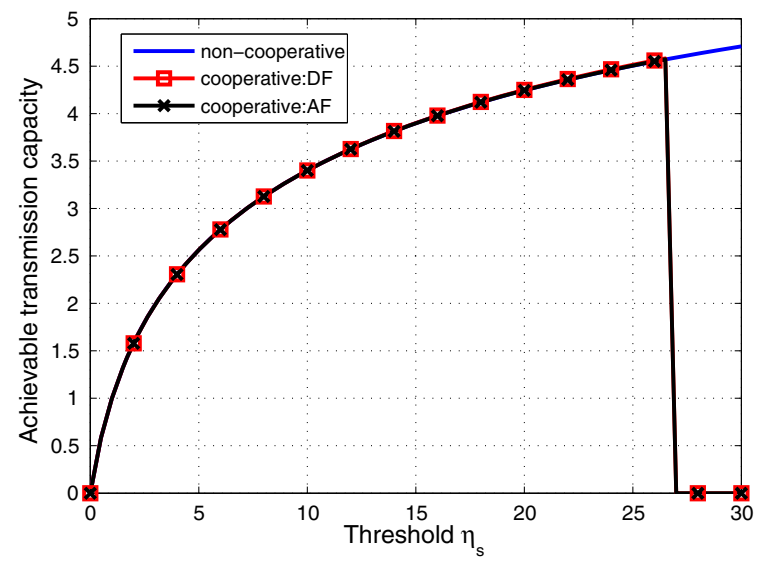

(c) $d_{i 0}=100, d_{00}=20$

Figure 8 The maximum achievable transmission capacity of the cognitive network when the distance between the PT and the PR varies. (a) $d_{i 0}=100, d_{00}=100$, (b) $d_{i 0}=100, d_{00}=50$ and (c) $d_{i 0}=100, d_{00}=20$. 
$\frac{I_{s 0}}{I_{s r}}$ and the power ratio $\gamma_{p s}$. Thus in this study, we fix the distance from the PT to the PR to be $100 \mathrm{~m}\left(d_{00}=100\right)$ and consider different power ratios $\left(\gamma_{p s}\right)$ and different interference ratios, with the latter achieved by changing the distances $\left(d_{i 0}\right)$ from ST1 and ST2 to the PR while

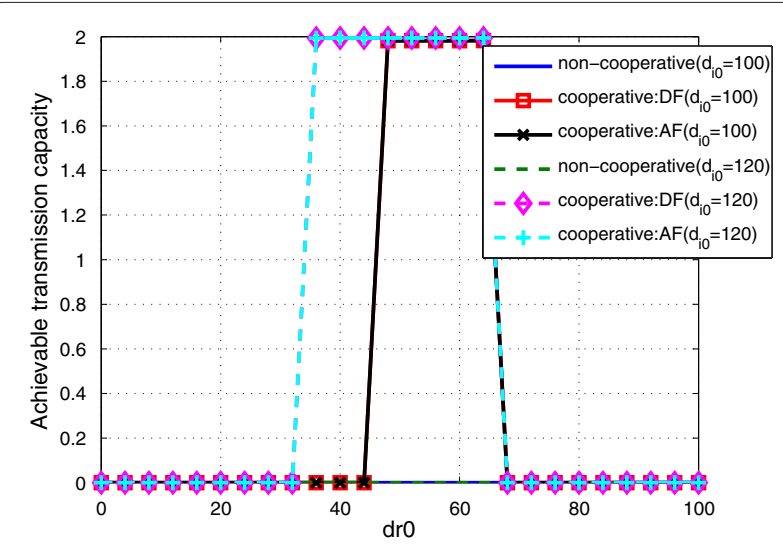

(a) $\gamma_{p s}=5, \beta=0$

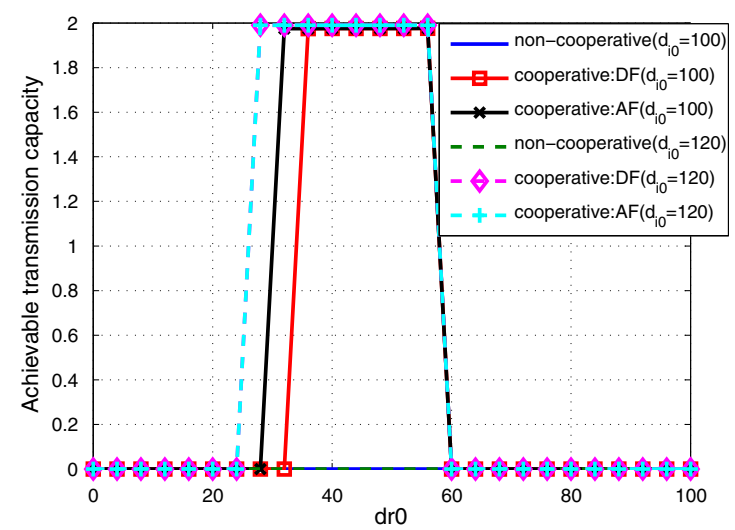

(b) $\gamma_{p s}=10, \beta=0$

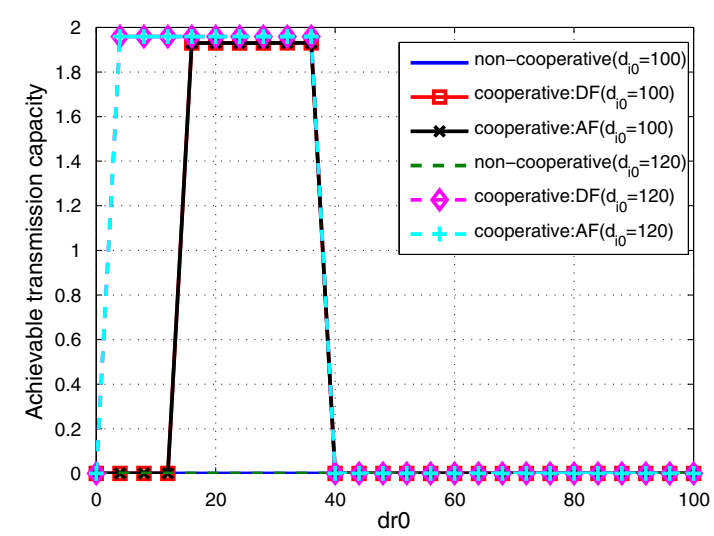

(c) $\gamma_{p s}=50, \beta=0$

Figure 9 Achievable transmission capacity of the cognitive network versus $\boldsymbol{d}_{\boldsymbol{r} \mathbf{0}}$ under variable $\gamma_{p s}$ and $\boldsymbol{d}_{\boldsymbol{i} \mathbf{0}}$ when $\beta=\mathbf{0}$.

(a) $\gamma_{p s}=5, \beta=0$ (b) $\gamma_{p s}=10, \beta=0$ and (c) $\gamma_{p s}=50, \beta=0$. keeping the ST1-ST2 line perpendicular to the PT-PR line as shown in Figure 3. The receiver threshold of the secondary network is set to be the same as that of the PR. Figures 9 and 10 report the results when $\beta=\left\{0, \frac{\pi}{3}\right\}$,

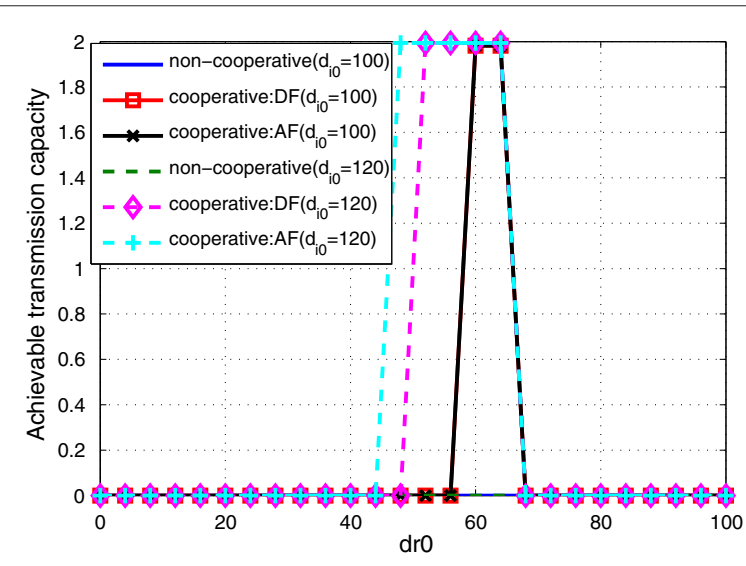

(a) $\gamma_{p s}=5, \beta=\frac{\pi}{3}$

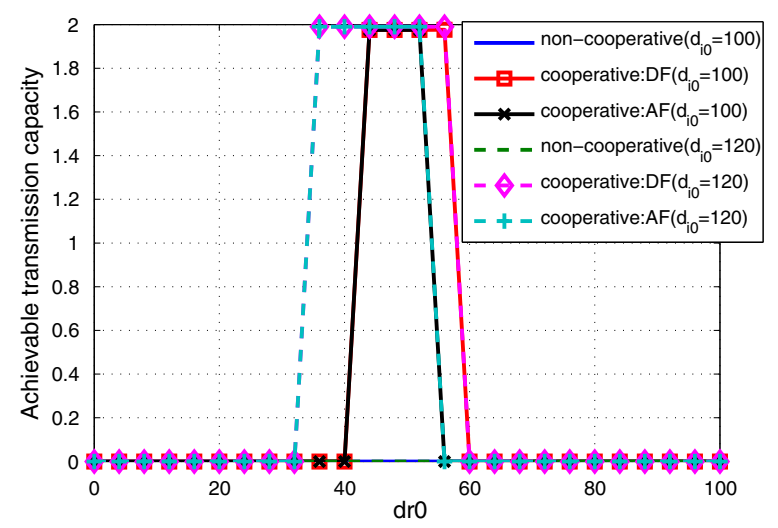

(b) $\gamma_{p s}=10, \beta=\frac{\pi}{3}$

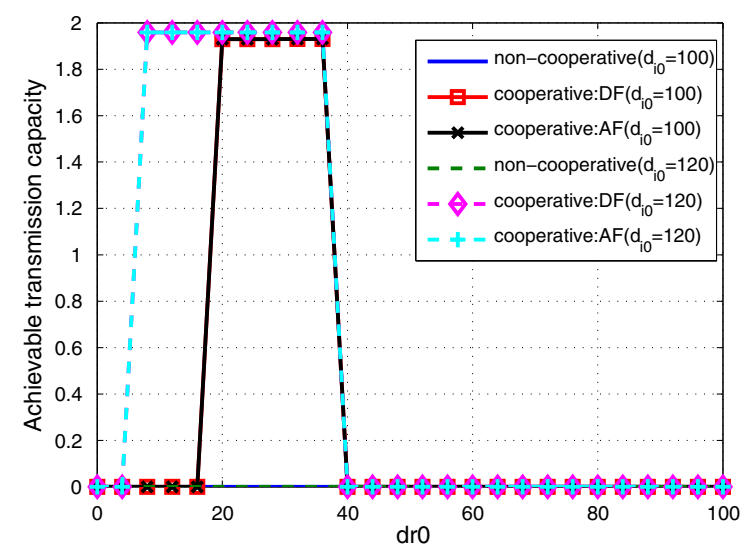

(c) $\gamma_{p s}=50, \beta=\frac{\pi}{3}$

Figure 10 Achievable transmission capacity of the cognitive network versus $\boldsymbol{d}_{\boldsymbol{r} 0}$ under variable $\gamma_{p s}$ and $\boldsymbol{d}_{i 0}$ when $\beta=\frac{\pi}{3}$. (a) $\gamma_{p S}=5, \beta=\frac{\pi}{3}$, (b) $\gamma_{p S}=10, \beta=\frac{\pi}{3}$ and (c) $\gamma_{p S}=50, \beta=\frac{\pi}{3}$. 
$\gamma_{p s}=\{5,10,50\}$, and $d_{i 0}=\{100,120\}$. Note that we have tested a full range of parameter settings of $\beta, \gamma_{p s}$, and $d_{i 0}$, and obtained very similar results.

We first observe that the cognitive radio network without providing cooperative relaying can not satisfy the outage probability constraints of the primary and the secondary systems for all parameter settings, thus achieving a zero capacity. Nevertheless, when cooperative relaying is adopted, the outage probability constraints can be satisfied and thus a nonzero capacity is achieved if the relay resides in the feasible location region. This is consistent with our results obtained from Figures 4, 5, 6, 7, and 8 . Second, we notice that the feasible location region of the relay becomes larger when $d_{i 0}$ is increased from 100 to $120 \mathrm{~m}$. This is because the interference ratio $\frac{I_{s 0}}{I_{s r}}$ increases when the neighboring SUs are moved far away, enlarging the feasible location region. For instance, when $\gamma_{p s}=5$ in Figure 9a, a nonzero capacity of around 2 is achieved as $d_{r 0}$ increases from 48 to $67 \mathrm{~m}$ when $d_{i 0}=100 \mathrm{~m}$ (the second and third curves), while for the same capacity value $d_{r 0}$ changes from 36 to $68 \mathrm{~m}$ when $d_{i 0}=120 \mathrm{~m}$ (the fifth and sixth curves). A similar conclusion can be drawn from other scenarios shown in Figures 9 and 10. Furthermore, by comparing Figure 9a,b,c, one can see that the feasible location region increases gradually when the power ratio $\gamma_{p s}$ changes from 5 to 50 . This is accordance with Equation 14, which indicates that a bigger $\gamma_{p s}$ provides a larger feasible location region. Figure 10 also demonstrates the same trend. Finally by comparing Figures 9 and 10 , we notice that the feasible range of $d_{r 0}$ corresponding to nonzero capacity is shorter when $\beta=\frac{\pi}{3}$ compared to the case of $\beta=0$; this is because the feasible location region of the relay is the overlapping area of the two disks centered at PT and PR, as shown in Figure 2. For example, when $\gamma_{p s}=5$ and $d_{i 0}=100 \mathrm{~m}$ (see Figures 9a and 10a), a capacity value of around 2 is obtained when $d_{r 0}$ changes from 48 to $67 \mathrm{~m}$ for $\beta=0$ while for the same nonzero capacity value $d_{r 0}$ changes from 58 to $66 \mathrm{~m}$ when $\beta=\frac{\pi}{3}$. These results are consistent with our previous analysis in Section 5 (see Equation 14).

\section{Conclusion}

In this paper, we investigate the achievable transmission capacity of a cooperative cognitive network under the outage probability constraints from both the primary and the secondary system. The probabilities of successful transmissions in the primary and the secondary network with or without cooperative relaying are derived based on the physical interference model. The maximum achievable transmission capacities of the secondary network with or without cooperative relaying in terms of bits $/ \mathrm{hop} / \mathrm{s} / \mathrm{Hz} /$ node are obtained based on Shannon's theory. Our numerical results indicate that cooperative relaying between the primary and the secondary system can help the secondary network to achieve a higher transmission capacity in some cases, and the capacity gain on cooperative relaying largely depends on the location of the relay, the power ratio, as well as the receiving threshold of the secondary network. For future research, we will consider more complicated cooperative cognitive network scenarios and more effective relay selection algorithms for cooperative cognitive networks.

\section{Competing interests}

The authors declare that they have no competing interests.

\section{Acknowledgements}

This work is supported by the National Natural Science Foundation of China (Grant No. 61172074, 61272505, and 61172074) and the National Science Foundation of the US (CNS-1162057, CNS-1265311, AST-1443858, and AST-1443916)

\section{Author details}

'School of Electronics and Information Engineering, Beijing Jiaotong University, No. 3 Shangyuancun, Haidian District, Beijing 100044, People's Republic of China. ${ }^{2}$ Department of Computer Science, The George Washington University, 2121 I St NW, Washington, DC 20052, USA. ${ }^{3}$ VTT Technical Research Centre of Finland, Kaitoväylä 1, Oulu, FI 02044, Finland. ${ }^{4}$ Department of Computer Science, University of Pittsburgh, 4200 Fifth Ave., Pittsburgh, PA 15260, USA.

Received: 28 August 2014 Accepted: 2 March 2015

Published online: 28 March 2015

\section{References}

1. JM Peha, Sharing spectrum through spectrum policy reform and cognitive radio. Proc. IEEE. 97(4), 708-719 (2009)

2. M Song, $C X$ in, $Y$ Zhao, $X$ Cheng, Dynamic spectrum access: from cognitive radio to network radio. IEEE Wireless Commun. 19(1), 23-29 (2012)

3. H Li, X Cheng, K Li, C Hu, N Zhang, W Xue, Robust collaborative spectrum sensing schemes for cognitive radio networks. IEEE Trans. Parallel Distributed Syst. 25(8), 2190-2200 (2014)

4. X Xing, $T$ Jing, $W$ Cheng, $Y$ Huo, $X$ Cheng, Spectrum prediction in cognitive radio networks. IEEE Wireless Commun. 20(2), 2190-2200 (2013)

5. W Zhou, T Jing, W Cheng, T Chen, Y Huo, in International Conference on Cognitive Radio Oriented Wireless Networks. Combinatorial auction based channel allocation in cognitive radio networks (Washington DC, USA, 2013), pp. 135-140

6. X Xing, T Jing, Y Huo, H Li, X Cheng, in IEEE INFOCOM. Channel quality prediction based on Bayesian inference in cognitive radio networks (Turin, Italy, 2013), pp. 1465-1473

7. J Lee, S Lim, JG Andrew, D Hong, in IEEE ICC. Achievable transmission capacity of secondary system in cognitive radio networks (Cape Town, South Africa, 2010), pp. 1-5

8. K Huang, VKN Lau, Y Chen, Spectrum sharing between cellular and mobile ad hoc networks: transmission-capacity trade-off. IEEE J. Select. Areas Commun. 27(7), 1256-1267 (2009)

9. C Li, H Dai, in IEEE INFOCOM. Transport throughput of secondary networks in spectrum sharing systems (Shanghai, China, 2011), pp. 2732-2740

10. T Jing, $X$ Chen, $Y$ Huo, $X$ Cheng, in IEEE INFOCOM. Achievable transmission capacity of cognitive mesh networks with different media access control (Orlando, USA, 2012), pp. 1764-1772

11. A Goldsmith, SA Jafar, I Maric, S Srinivasa, Breaking spectrum gridlock with cognitive radios: an information theoretic perspective. Proc. IEEE. 97(5), 894-914 (2009)

12. PJ Kolodzy, in Proceedings of SDR Forum. Cognitive radio fundamentals (Singapore, 2005)

13. SP Weber, X Yang, JG Andrews, Transmission capacity of wireless ad hoc networks with outage constraint. IEEE Trans. Inform. Theory. 51(12), 4091-4102 (2005) 
14. C Yin, L Gao, T Liu, S Cui, in IEEE ICC. Transmission capacities for overlaid wireless ad hoc networks with outage constraints (Dresden, Germany, 2009), pp. 1-5

15. C Chang, J Chang, Optimal design parameters in a multihop packet radio network using random access techniques. Comput. Netw. ISDN Syst. 11(5), 337-351 (1986)

16. $\mathrm{H} \mathrm{Li}, \mathrm{X}$ Cheng, $\mathrm{K} \mathrm{Li}, \mathrm{X}$ Xing, $\mathrm{T}$ Jing, in IEEE INFOCOM Mini-Conference. Utility-based cooperative spectrum sensing scheduling in cognitive radio networks (Turin, Italy, 2013), pp. 165-169

17. R Zhang, J Zhang, Y Zhang, C Zhang, in IEEE INFOCOM. Secure crowdsourcing-based cooperative spectrum sensing (Turin, Italy, 2013), pp. 2526-2534

18. S Li, Z Zheng, E Ekici, N Shroff, in IEEE INFOCOM. Maximizing system throughput by cooperative sensing in cognitive radio networks (Orlando, USA, 2012), pp. 1575-1583

19. XXing, $T$ Jing, W Cheng, Y Huo, X Cheng, T Znati, Cooperative spectrum prediction in multi-PU multi-SU cognitive radio networks. ACM/Springer Mobile Netw. Appl. 19(4), 502-511 (2014)

20. O Simeone, Y Bar-Ness, U Spagnolini, Stable throughput of cognitive radios with and without relaying capability. IEEE Trans. Commun. 55(12), 2351-2360 (2007)

21. S Kompella, GD Nguyen, JE Wieselthier, in IEEE INFOCOM. Stable throughput tradeoffs in cognitive shared channels with cooperative relaying (Shanghai, China, 2011), pp. 1961-1969

22. T Jing, $\mathrm{S} Z \mathrm{hu}, \mathrm{H} \mathrm{Li}$, X Cheng, Y Huo, in IEEE INFOCOM Mini-Conference. Cooperative relay selection in cognitive radio networks (Turin, Italy, 2013), pp. 175-179

23. W Li, X Cheng, $T$ Jing, $X$ Xing, in IEEE INFOCOM. Cooperative multi-hop relaying via network formation games in cognitive radio networks (Turin, Italy, 2013), pp. 995-1003

24. J Zhang, Q Zhang, in ACM MobiHoc. Stackelberg game for utility-based cooperative cognitive radio networks (New Orleans, USA, 2009), pp. 23-31

25. C Kam, S Kompella, GD Nguyen, JE Wieselthier, in IEEE INFOCOM Mini-Conference. Multicast throughput stability analysis for cognitive cooperative random access (Turin, Italy, 2013), pp. 170-174

26. L Guo, X Ding, H Wang, Q Li, Cooperative relay service in a wireless lan. IEEE J. Selected Areas Commun. 25(2), 355-368 (2007)

27. X Cheng, D-Z Du, L Wang, B Xu, Relay sensor placement in wireless sensor networks. Wireless Netw. 14(3), 347-355 (2008)

28. B Wang, J Zhang, in IEEE INFOCOM. Throughput scaling of wideband sensory relay networks: cooperative relaying, power allocation and achievable rates (Barcelona, Spain, 2006), pp. 1-12

29. X Wang, W Huang, S Wang, J Zhang, C Hu, Delay and capacity tradeoff analysis for motioncast. IEEE/ACM Trans. Netw. 19(5), 1354-1367 (2011)

30. P Gupta, PR Kumar, The capacity of wireless networks. IEEE Trans. Inform. Theory. 46(2), 388-404 (2000)

31. C Yin, L Gao, S Cui, Scaling laws for overlaid wireless networks: a cognitive radio network vs. a primary network. IEEE/ACM Trans. Netw. 18(4), 1317-1329 (2010)

32. S-W Jeon, N Devroye, M Vu, S-Y Chung, V Tarokh, Cognitive networks achieve throughput scaling of a homogeneous network. IEEE Trans. Inform. Theory. 57(8), 5103-5115 (2011)

33. W Huang, X Wang, Capacity scaling of general cognitive networks. IEEE/ACM Trans. Netw. 20(5), 1505-1513 (2011)

34. K Hong, Y Hua, Throughput analysis of large wireless networks with regular topologies. EURASIP J. Wireless Commun. Netw. 2007(1), 3-3 (2007)

35. JN Laneman, DNC Tse, GW Wornell, Cooperative diversity in wireless networks: Efficient protocols and outage behavior. IEEE Trans. Inform. Theory. 50(12), 3062-3080 (2004)

36. J Lee, H Wang, JG Andrew, D Hong, Outage probability of cognitive relay networks with interference constraints. IEEE Trans. Wireless Commun. 10(2), 390-395 (2011)

\section{Submit your manuscript to a SpringerOpen ${ }^{\circ}$ journal and benefit from:}

- Convenient online submission

Rigorous peer review

- Immediate publication on acceptance

- Open access: articles freely available online

- High visibility within the field

- Retaining the copyright to your article

Submit your next manuscript at $\boldsymbol{\nabla}$ springeropen.com 